\title{
Editorial
}

\section{Urinary lithiasis: the perfect balance}

\section{Litíase urinária: a busca do ponto de equilíbrio}

Fernando Korkes ${ }^{1,2}$

Urinary lithiasis is one of the conditions that have undergone revolutionary changes in treatment over the past decades. The technological advances in diagnosis (with ultrasound and computed tomography) and interventional treatment of urinary calculi observed in the 1980s and 1990s were very striking. In this century, we have seen the improvement of techniques and the refinement of indications. Currently, the treatment of urinary calculi is almost exclusively by minimally invasive procedures, with extremely low rates of severe complications.

However, having minimally invasive procedures available does not mean they should be used indiscriminately. Many physicians sworn the Hippocratic Oath stating "they will not cut through the bladder", but this is part of a distant past, and has been efficiently replaced by less invasive procedures. The principle of primum non nocere [first do not harm] remains a maxim of the medical profession. Therefore, the most appropriate interventions will be those precisely indicated for each situation. In this context, some situations are extremely commonplace, such as the following.
Renal colic is a common cause of patient visits to the emergency room, and ureterolithiasis is the most common diagnosis in these cases. One of the main precautions in these situations is, on one hand, to avoid the excessive indication of surgical procedures for calculi likely to be spontaneously eliminated without further damage. In this sense, professionals involved in treating these patients should maintain an ethical position, educating and reassuring patients, without letting themselves be led by financial interests, and focusing on ensuring the best therapeutic outcomes for patients. On the other hand, another important step is being careful not to take the disease for granted, which could result in undertreatment of patients' pain, missed diagnosis of infections associated with the urinary tract obstruction (which could aggravate and impair renal function), or not appropriately following up patients care. The role of the physician at this time includes avoiding complications and loss of renal function, and guiding followup care for prevention of new episodes. The constant training and updating of urgency care professionals is essential in this context. Decision-making cannot be

${ }^{1}$ Faculdade de Medicina do ABC, Santo André, SP, Brazil.

${ }^{2}$ Hospital Israelita Albert Einstein, São Paulo, SP, Brazil.

Corresponding author: Fernando Korkes - Avenida Albert Einstein, 627/701 - room 419 - Morumbi - Zip code: 05652-000 - São Paulo, SP, Brazil Phone: (55 11) 3884-2233 - E-mail: fkorkes@terra.com.br

DOI: 10.1590/S1679-45082015ED3312 
merely based on the size of the calculus, which is often the only parameter taken into consideration. Topography in the ureter, disease duration, hydronephrosis, history of past calculus elimination, pain intensity, patient age and social factors must also always be considered. The alpha-blockers currently available to increase the chance of eliminating calculi are highly efficient and should be prescribed whenever possible. However, the limitations of the off-label use of these drugs and their side effects must be well known. The double-J stents used in many of these patients are revolutionary for reducing the incidence of complications, but they can also cause much discomfort to some patients..$^{(1,2)}$

In cases of asymptomatic calculi, the nephrolithiasis diagnosis is often made based on tests performed routinely or for other reasons. What to do with these calculi is a common question for clinicians, surgeons and their patients. Unnecessary interventions should be avoided, but we should not stop treating calculi with simple measures when the lack of timely treatment may lead to the requirement for more complex procedures. For staghorn calculi, there is little doubt. Historical studies not likely to be reproduced for ethical reasons demonstrated a $30 \%$ rate of mortality for these calculi when untreated. ${ }^{(3)}$ Calyceal stones, in turn, are more controversial. While calculi of about $10 \mathrm{~mm}$ progress in $77 \%$ of cases and require intervention in $26 \%$ of cases within up to 3 years, for calculi of about $4 \mathrm{~mm}$ in lower calyces, watchful waiting seems to be the best alternative. ${ }^{(4)}$ In the only prospective randomized trial comparing watchful waiting versus extracorporeal shockwave lithotripsy (ESWL), over a period of 2.2 years, watchful waiting was associated with a higher rate of urgency interventions ( $8.7 \%$ versus $0 \%$ ). However, quality of life did not seem to change according to the authors. ${ }^{(5)}$ For calyceal calculi $<10 \mathrm{~mm}$, there is no current consensus on whether or not and when to intervene. ${ }^{(6)}$

It is worth noting that it is very important to consider the individual aspects of the patient when making decisions. A 1-cm calculus in the lower calyx, stable for 10 years, in a 80 -year old patient is different from a 1 -cm stone in the upper calyx, in a 30 -year old patient with cystinuria and recurrent calculus formation. Some social factors, such as frequent travelers, airline pilots, individuals who live far from hospitals, with a solitary kidney, history of renal colics, women wanting future pregnancies etc., must be taken into consideration when deciding about management. ${ }^{(2,7)}$

Regarding treatment modalities, there is a clear tendency towards changing the way urinary stones have been treated so far. Open surgeries have been virtually abandoned in recent decades. ESWL appeared in the 1980s and has been indicated for a large number of cases. ${ }^{(8)}$ With the development and increasing use of endourological procedures, particularly percutaneous renal surgery and ureteroscopy, a global trend towards reduction in the number of indications for ESWL has been observed. With the emergence of flexible ureterorenoscopy, many renal and proximal ureteral calculi started being treated with this delicate and expensive equipment. ${ }^{(1,6)}$

Another extremely important point to be considered is the recurrence of lithiasis. If nothing is done, the recurrence rate for urolithiasis is estimated at $50 \%$ at 5 years. ${ }^{(9)}$ In addition to the inconvenience to patients, the costs to the healthcare system are extremely high. ${ }^{(10)}$ Relatively simple assessments can be used to help preventing this multifactorial disease, which is particularly important for individuals with a history of multiple calculi, large calculi, childhood calculi or a solitary kidney. Dietary and behavioral factors can be corrected, mainly by increasing water intake, which significantly decreases the risk of relapse. Laboratory evaluation based on serum and 24-hour urine parameters, and crystallographic analysis of previously eliminated/ removed urinary calculi when required are also extremely important in these cases. Urolithiasis can also result from diseases in other organs, of which the greatest example is primary hyperparathyroidism. It can also help identify the type of metabolic disorder presented by the patient, thus impacting the preventive treatment, patient guidance and even future treatments in case new calculi appear. Different drugs may be used to objectively prevent recurrence, such as thiazides for idiopathic hypercalciuria, citrate for hypocitraturia, 
allopurinol for hyperuricosuria, captopril and/or tiopronin for cystinuria. ${ }^{(9)}$

Urinary lithiasis, as one of the oldest and most prevalent conditions in humans, has undergone a complete revolution in terms of understanding and management in recent decades. It is up to us, physicians, to know how to correctly balance the use of diagnostic and therapeutic methods, as well as to resolve any potential conflicts of interests, always acting in the best interest of our patients and, ultimately, of society as a whole.

\section{REFERENCES}

1. Bader MJ, Eisner B, Porpiglia F, Preminger GM, Tiselius HG. Contemporary management of ureteral stones. Eur Urol. 2012;61(4):764-72. Review.

2. Korkes F, Gomes SA, Heilberg IP. Diagnóstico e tratamento de litíase ureteral. J Bras Nefrol. 2009;31(1):55-61.
3. Rous SN, Turner WR. Retrospective study of 95 patients with staghorn calculus disease. J Urol. 1977;118(6):902-4.

4. Burgher A, Beman M, Holtzman JL, Monga M. Progression of nephrolithiasis: long-term outcomes with observation of asymptomatic calculi. J Endourol. 2004;18(6):534-9.

5. Keeley FX Jr, Tilling K, Elves A, Menezes P, Wills M, Rao N, et al. Preliminary results of a controlled trial of prophylactic shock wave lithotripsy for small asymptomatic renal calyceal stones. BJU Int. 2001;87:1-8.

6. Tiselius HG, Alken P, Buck C, Galluci M, Knoll T, Sarica K, et al. Guidelines on urolithiasis. The Netherlands: European Association of Urology; 2008.

7. Korkes F, Rauen EC, Heilberg IP. Urolithiasis and pregnancy. J Bras Nefrol. 2014;36(3):389-95.

8. Chaussy C, Schmiedt E. Extracorporeal shock wave lithotripsy (ESWL) for kidney stones. An alternative to surgery? Urol Radiol. 1984;6(2):80-7.

9. Heilberg IP, Schor N. Renal stone disease: Causes, evaluation and medical treatment. Arq Bras Endocrinol Metabol. 2006;50(4):823-31. Review.

10. Korkes F, Silva II JL, Heilberg IP. Costs for in hospital treatment of urinary lithiasis in the Brazilian public health system. einstein (São Paulo). 2011; 9(4 Pt 1):518-22. 